\title{
Diaphragmatic myoclonus due to SARS-CoV-2 infection
}

\author{
Barbara Borroni ${ }^{1,2}$ (1) - Stefano Gazzina ${ }^{1} \cdot$ Fedele Dono $^{3} \cdot$ Valentina Mazzoleni $^{1} \cdot$ Paolo Liberini $^{1} \cdot$ Claudia Carrarini $^{3}$. \\ Mirella Russo $^{3} \cdot$ Michela Pontolillo $^{4}$. Jacopo Vecchiet ${ }^{4} \cdot$ M. Onofrj ${ }^{3} \cdot$ Laura Bonanni $^{3}$
}

Received: 12 July 2020 / Accepted: 24 September 2020 / Published online: 22 October 2020

(C) Fondazione Società Italiana di Neurologia 2020

\begin{abstract}
A wide range of neurological signs and symptoms have been associated with SARS-CoV-2 infection. In the present report, we described two Italian patients diagnosed with diaphragmatic myoclonus after COVID-19. In both cases, mild lymphocytosis at cerebrospinal fluid analysis and no structural brain changes were reported. The pathophysiological origin of the myoclonus in the two cases was different. In case 1, electroencephalogram did not reveal any cortical correlates and brain imaging of the spine was unremarkable, while in case 2, cortical origin of myoclonus was demonstrated. With the present two cases, we confirm and extend the neurological manifestations of SARS-CoV-2 infection.
\end{abstract}

Keywords Myoclonus $\cdot$ Diaphragmatic $\cdot$ SARS-CoV-2 $\cdot$ COVID-19 $\cdot$ Neurology

\section{Introduction}

The 2019 new coronavirus (SARS-CoV-2) is a respiratory virus which has become increasingly prevalent worldwide, reaching a pandemic stage on March 2020 according to WHO, with more than 6 million people affected at 1 st June, according to WHO data. The clinical manifestations of SARS-CoV-2 include fever, dry cough, fatigue, headache, gastrointestinal discomfort, dyspnea, muscle ache, and a severe pneumonia causing respiratory distress [1]. Neurological signs in patients with SARS-CoV-2 have been widely reported, suggesting a neuroinvasive nature of virus $[2,3]$.

Electronic supplementary material The online version of this article (https://doi.org/10.1007/s10072-020-04766-y) contains supplementary material, which is available to authorized users.

Barbara Borroni

bborroni@inwind.it

1 Neurology, Department of Clinical and Experimental Sciences, University of Brescia, Brescia, Italy

2 Clinica Neurologica, Università degli Studi di Brescia, P.le Spedali Civili 1, 25123 Brescia, Italy

3 Department of Neuroscience, Imaging and Clinical Sciences, University G. d'Annunzio of Chieti-Pescara, Chieti, Italy

4 Department of Medicine and Aging Sciences, University G. d'Annunzio of Chieti-Pescara, Chieti, Italy
With the present observation, we report two cases of diaphragmatic myoclonus as neurological subacute manifestation of SARS-CoV-2 infection.

Case 1 A 54-year-old woman with SARS-CoV-2 presented on March 16, 2020, to the emergency room in Brescia, one of the epicenter of SARS-CoV-2 infection in Italy [4], with fever, dry cough, and dyspnea. Thoracic ultrasound was consistent with the suspicion of viral pneumonia, but the nasopharyngeal swab resulted negative. The initial treatment was supportive, and azithromycin was added as empirical therapy. However, in the following 2 weeks, fever and dry cough persisted and she started complaining episodic chest pain, associated with involuntary rapid contractions of the abdominal wall, especially at night. Her past medical history was unremarkable except for hypertension.

For these symptoms, on April 1, 2020, she returned to the emergency room. The body temperature was $36.8^{\circ} \mathrm{C}\left(98{ }^{\circ} \mathrm{F}\right)$, and oxygen saturation was $95 \%$ at room air. Myocardial infarction was excluded. Nasopharyngeal swab for SARS-CoV-2 was repeated and she tested positive. She began to receive treatment with hydroxychloroquine and low molecular weight heparin.

On May 7, 2020, the patient was admitted to the Neurological Unit for the persistency of the jerky contractions of abdominal muscles, causing disabling shortness of breath, despite a complete recovery of fever and cough. On examination, she presented with paroxysmal involuntary movements of her abdomen, lasting a few minutes, and presenting from 10 to 15 times a day (see supplementary video 1 ). The 
movements did not affect her chewing or swallowing, nor impact her bowel function. They were not provoked or worsened by any positions or triggered by any maneuvers. Moreover, not disabling, small-amplitude twitches of left limbs on maintaining a posture were observed.

Electromyographic recording confirmed rhythmic and synchronous contractions of the diaphragm at $3-\mathrm{Hz}$ frequency as the cause of her abdominal movements (see Fig. 1), thus making the diagnosis of diaphragmatic myoclonus or van Leeuwenhoek's disease [5]. Phrenic nerve conduction was normal bilaterally, as well as magnetic resonance imaging of the head and spine, electroencephalogram (EEG), somatosensory evoked potentials, and cerebrospinal fluid analysis. Cerebrospinal fluid (CSF) evaluation showed mild lymphocytosis $\left(8 \mathrm{cell} / \mathrm{mm}^{3}\right)$ with normal level of glucose and proteins. Clonazepam was titrated with significant benefit $(0.5 \mathrm{mg}$ tid $)$.

Case 2 An 80-year-old man was admitted on May 10, 2020, to the emergency room in Chieti due to mild dyspnea, persistent fever $\left(38^{\circ} \mathrm{C}\right)$, and dry coughing. His past medical history was unremarkable. Arterial blood gas analysis showed normal blood oxygen saturation $\left(\mathrm{SO}_{2}=95.6 \%\right)$, mild hypoxemia $(71.9 \mathrm{mmHg})$, hypocapnia $(22.1 \mathrm{mmHg})$, and alkalosis $(\mathrm{pH}$
$=7.534)$. Thoracic computer tomography scan showed a ground-glass nodule at the left apical pulmonary lobe segment. Oropharyngeal and nasopharyngeal swabs supported the diagnosis of SARS-CoV-2. The patient was then empirically treated with azithromycin and supportive therapy with a relief of the symptoms in 10 days with no need of ventilation throughout the entire period.

On June 2, 2020, jerky myoclonic contractions of the diaphragm appeared, causing disabling shortness of breath, despite the complete recovery of previous respiratory symptoms (see supplementary video 2). Radiography of the thorax showed a complete resolution of the pulmonary infection. Patient appeared conscious and collaborative, although was not well oriented in space and time. Computed tomography brain scan did not show any acute brain abnormalities. The EEG recording showed lateralized periodic discharges (LPDs) synchronous and asynchronous with the diaphragmatic myoclonic movements (see Fig. 2A). CSF evaluation showed lymphocytosis $\left(24 \mathrm{cell} / \mathrm{mm}^{3}\right)$ with normal level of glucose and slight increase of proteins $(46.2 \mathrm{mg} / \mathrm{dl})$. Polymerase chain reaction (PCR) for SARS-CoV-2 as well as herpes viruses, cytomegalovirus, Epstein-Barr

A

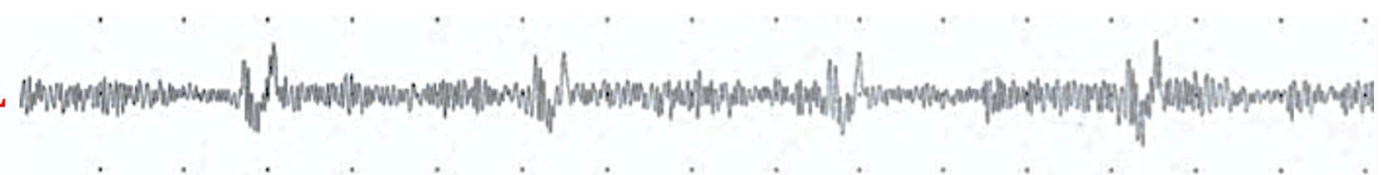

B
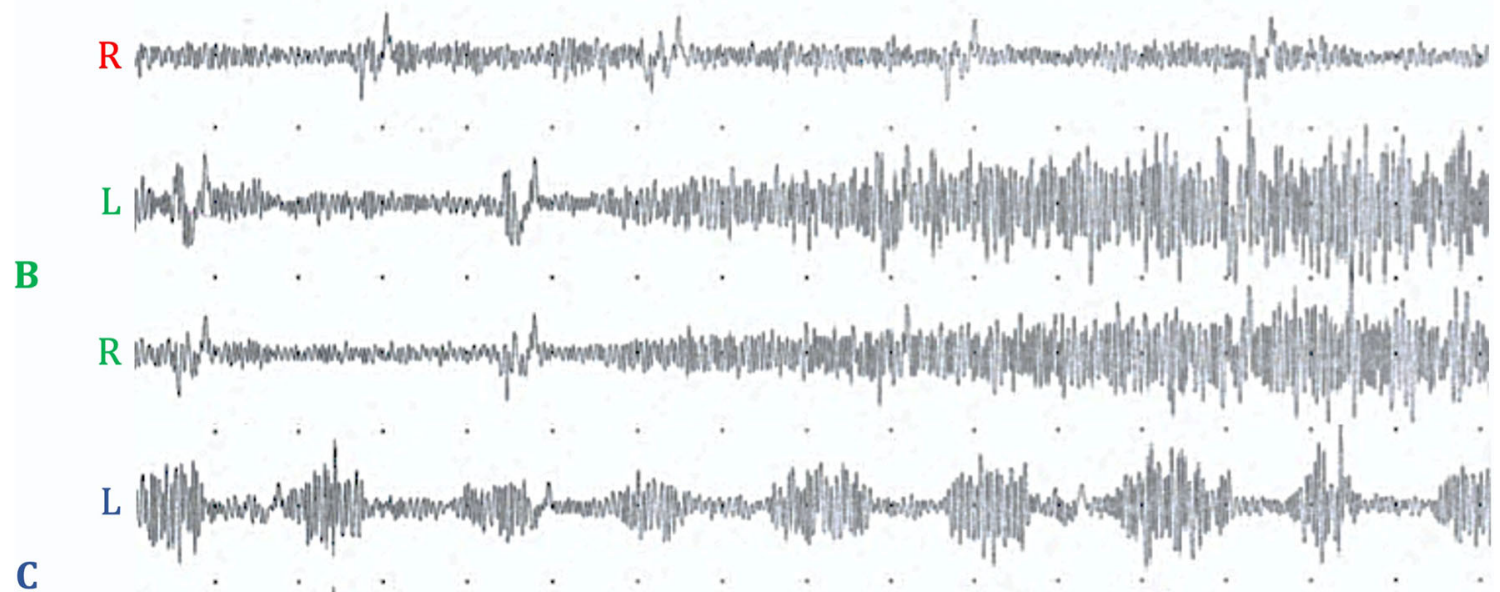

C

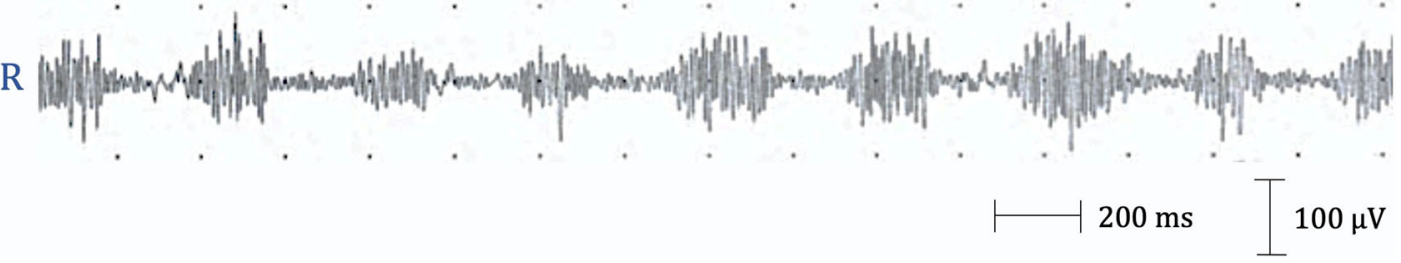

Fig. 1 Surface recording of left and right hemidiaphragms in case 1. A Patient at rest, ECG artifact. B Deep inspiration. C Threehertz rhythmic synchronous discharges during involuntary contraction of abdominal muscles. Electrophysiological study of the phrenic nerve was performed by placing one monopolar needle (active electrode) two fingerbreadths above the xiphoid process and two monopolar needles (reference electrodes) over the anterior costal margin $16 \mathrm{~cm}$ from the active electrode. The same electrodes were used to derive electromyography activity of the diaphragm. L, left; R, right 
virus, and varicella zoster virus in the CSF was negative. Treatment with levetiracetam $(1000 \mathrm{mg}$ bid $)$ was started with resolution of the myoclonus and improvement of the EEG features after 3 days (see Fig. 2B).

\section{Discussion}

Diaphragmatic myoclonus is a rare condition and has been associated with many central and peripheral disorders including, but not limited to, encephalitis, stroke, osmotic demyelination, metabolic abnormalities, trauma, and phrenic nerve irritation, its etiology being still debated [6]. In our cases, the temporal sequence suggests, but does not prove, that SARS$\mathrm{CoV}-2$ was a causal factor of diaphragmatic myoclonus and cannot address the pathogenetic mechanism leading to the clinical picture. However, a number of similar cases are described in the literature where isolated myoclonus developed following viral infections of the upper respiratory tract or following an influenza-like illness, with no evidence of structural damage of the central nervous system [7].

Myoclonus can be a clinical feature of many infectious encephalitis and was notable in the acute and chronic phases of epidemic encephalitis lethargica, which arose almost

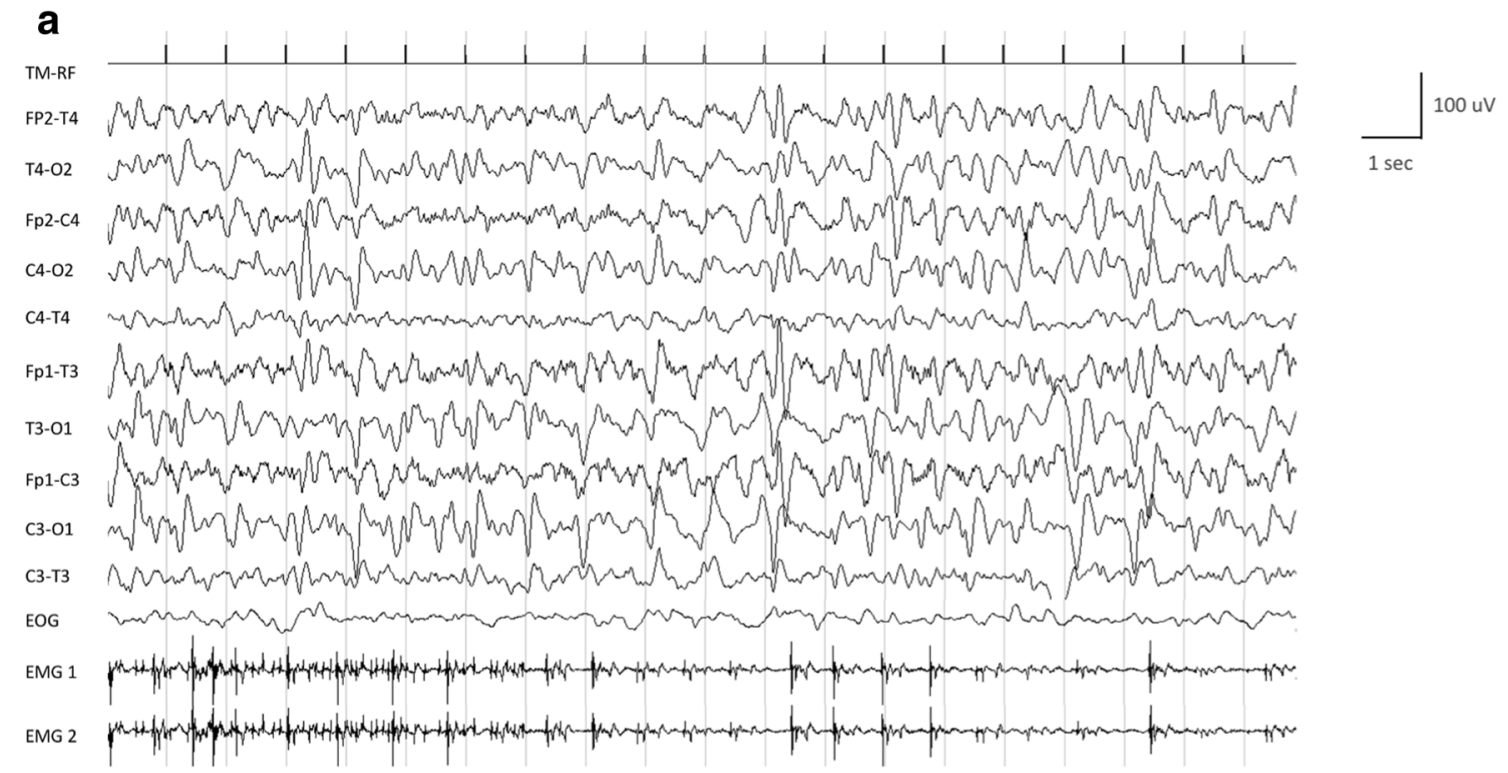

b

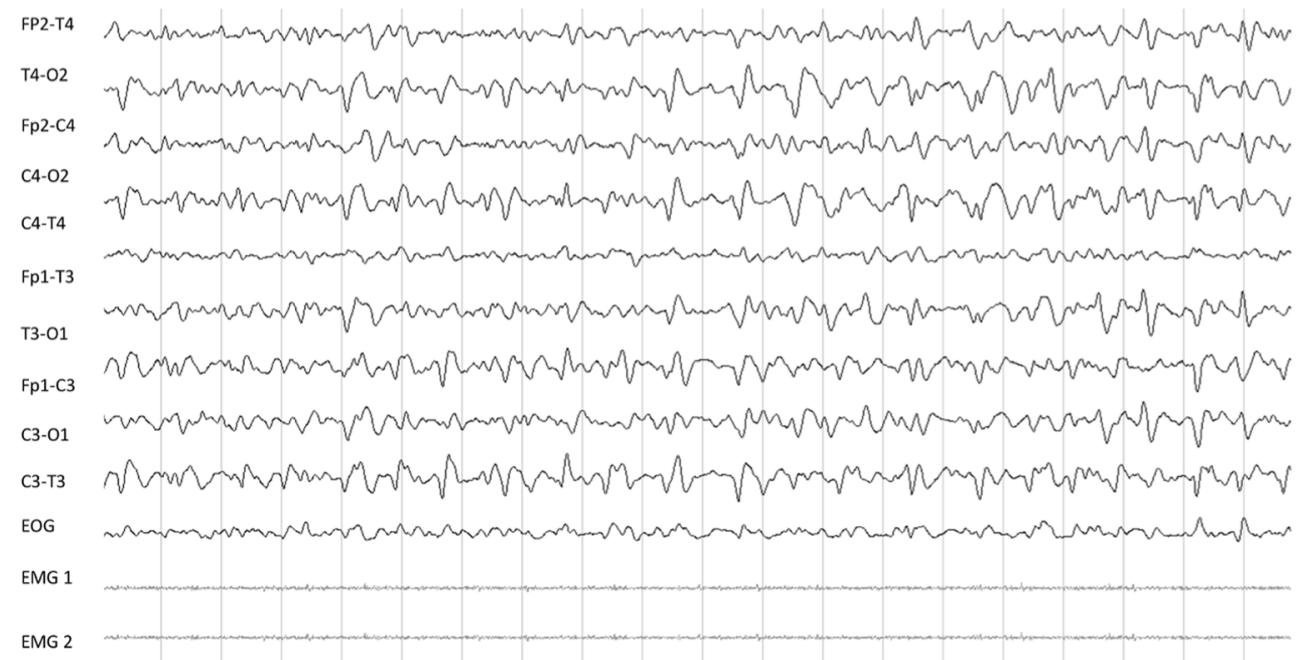

Fig. 2 Baseline (A) and follow-up (B) electroencephalogram (EEG) recording with surface electromyography (EMG) recording of case 2. A Baseline EEG showing lateralized periodic discharges (diffuse spike and spike-and-slow-wave complexes at $2-3 \mathrm{~Hz}$ and $100-150 \mu \mathrm{V}$ of amplitude) synchronous and asynchronous with diaphragm myoclonic jerks shown in surface EMG recording. B Follow-up EEG after levetiracetam
$1000 \mathrm{mg}$ twice daily showing diffuse spike and spike-wave complexes with a clear change in amplitude (up to $90 \mu \mathrm{V}$ ) and frequency $(1-2 \mathrm{~Hz}$ ). No myoclonic jerk at diaphragm has been detected as shown in the surface EMG recording. EOG, electro-oculogram; EMG1, right hemidiaphragm electrode; EMG2, left hemidiaphragm electrode 
contemporaneously with the 1918 Spanish influenza outbreak, caused by H1N1 virus [8]. Although the etiology of encephalitis lethargica remains uncertain, the Spanish flu virus has been implicated in the pathogenesis. Segmental myoclonus following herpes zoster infection is also well known and is usually transient [9].

Here, we draw attention to two patients who developed focal diaphragmatic myoclonus after SARS-CoV-2 infection. In our two cases, the diaphragmatic myoclonus was not accompanied by evidence of structural damage of the central nervous system. The pathophysiological origin of the myoclonus in our two cases is likely different. In case 1, EEG did not reveal any cortical correlates suggesting that the myoclonus was probably not of cortical origin. In case 2, EEG showed LPDs synchronous and asynchronous with the diaphragmatic myoclonic movements, with good response to levetiracetam. By looking at the time interval between cortical alterations and myoclonic jerks, these appear to be irregular (i.e., the cortical abnormalities were not invariably corresponding in time to myoclonic jerks); therefore, as a spinal myoclonus can be ruled out, a subcortical origin of the myoclonus cannot be excluded, even though the good response to levetiracetam would suggest an at least partial involvement of the cortex. This EEG pattern might be related to a widespread inflammatory effect due to SARS-CoV-2 CNS infection. In line with previous reports in the literature [10], even though PCR for SARS-CoV-2 performed on CSF was negative, we could not exclude the role of SARS-CoV-2 in the genesis of the cerebral inflammation.

It appears that myoclonus associated with SARS-CoV-2 infection is a heterogenous entity and that different sites of the central nervous system can be affected by the virus. Indeed, three cases of generalized myoclonus due to SARSCoV-2 infection have been recently reported [10].

With the present report of two cases, we confirm and extend the neurotropic properties of SARS-CoV-2 virus and point out to a further neurological clinical manifestation of the infection.

\section{Compliance with ethical standards}

Conflict of interest The authors declare that they have no conflict of interest.

Ethics approval The work was approved by local ethic committees of Brescia Hospital and Chieti Hospital.

Consent to participate Patients signed the informed consent to participate.

\section{References}

1. Xu YH, Dong JH, An WM et al (2020) Clinical and computed tomographic imaging features of novel coronavirus pneumonia caused by SARS-CoV. J Infect 8:394-400

2. Ma L, Jin H, Wang M et al (2020) Neurologic manifestations of hospitalized patients with coronavirus disease 2019 in Wuhan, China. JAMA Neurol 77:1-9

3. Das G, Mukherjee N, Ghosh S (2020) Neurological insights of COVID-19 pandemic. ACS Chem Neurosci 11:1206-1209

4. Benussi A, Pilotto A, Premi E et al. (2020) Clinical characteristics and outcomes of inpatients with neurologic disease and COVID-19 in Brescia, Lombardy, Italy. Neurology in press

5. Larner AJ (2005) Antony van Leeuwenhoek and the description of diaphragmatic flutter (respiratory myoclonus). Mov Disord 20: 917-918

6. Walton D, Bonello M, Steiger M (2018) Diaphragmatic flutter. Pract Neurol 18:224-226

7. Bathia K, Thomson D, Marsden CD (1992) Isolated postinfectious myoclonus. J Neurol Neurosurg Psychiatry 55:1089-1091

8. Ravenhol RT, Foege WH (1982) 1918 influenza, encephalitis lethargica, parkinsonism. Lancet 8303:860-864

9. Estraneo A, Saltalamacchia AM, Loreto V (2007) Spinal myoclonus following herpes zoster radiculitis. Neurology 68

10. Pezzini A, Padovani A (2020) Lifting the mask on neurological manifestations of COVID-19 Nat Rev Neurol 1-9. doi:https://doi. org/10.1038/s41582-020-0398-3

Publisher's note Springer Nature remains neutral with regard to jurisdictional claims in published maps and institutional affiliations. 